\title{
Prevention of mother to child transmission of HIV/AIDS in Eritrea: the Eritrean experience
}

\author{
Tadesse Teclebirhan', Araia Berhane², Jacob Mufunda' ${ }^{1}$ Andemariam Gebremichael ${ }^{1}$ \\ Institutional Affiliation \\ 'Orotta School of Medicine, Asmara, Eritrea \\ ${ }^{2}$ National Aids and Tuberculosis Control Department, Ministry of Health, Asmara, Eritrea
}

Correspondence to be sent to: Tadesse Teclebirhan MD Orotta School of Medicine P.O.Box 10549

Asmara, Eritrea

Email: tadesse09@gmail.com

Background: DThe combined risk of mother to child transmission of HIV/AIDS which is estimated at more than one third of live deliveries can be reduced to $<2 \%$ through antiretroviral prophylaxis. The study was done to determine the effectiveness of prevention of mother to child transmission (PMTCT) of HIV/AIDS in Eritrea where skilled care delivery is less than $30 \%$.

Objective: The objective of this study was to assess the use of $\mathrm{IMCl}$ guidelines in children presenting with diarrhea at Ghindae Hospital.

Methods: The retrospective study analyzed medical records of pregnant HIV positive women and their children before the introduction of PMTCT in 2002, while the prospective study followed up children born to pregnant HIV positive women who received PMTCT from 2006 to 2008.

Findings: : Twenty seven and $4.8 \%$ of the children born to HIV positive women prior to and after PMTCT were HIV positive when tested for HIV using ELISA at 18 months.

Conclusion: Health promotion targeted at scaling up skilled care delivery attendance can further reduce childhood morbidity and mortality from HIV/AIDS.

Keywords: PMTCT, Antiretroviral drugs, skilled care delivery, counseling.

\section{Introduction}

Prevention of mother to child transmission (PMTCT) of HIV/AIDS is a widely used strategy worldwide to prevent mother-to-child transmission of HIV that involves use of antiretroviral drugs, safe delivery practices, infant feeding, counseling, and support. ${ }^{1}$

Vertical transmission is one of the most important routes of HIV-1 transmission in sub-Saharan Africa responsible for more than $5 \%$ of childhood cases of HIV/AIDS. ${ }^{2}$ Since the WHO recommended the use of nevirapine as the standard prevention of mother to child transmission of HIV/AIDS during delivery using specific guidelines, the rates of vertically transmission of HIV/ AIDS has been decimated to less than $2 \%$ of babies born to HIV positive mothers in developed countries since this program became available in the late-1990s. ${ }^{3}$ PMTCT programs based on the administration of a single dose of nevirapine (NVP) to the mother and her baby at delivery have been adopted by many African countries as part of their national PMTCT policies and guidelines. ${ }^{4}$

PMTCT in Eritrea was adopted from WHO guidelines which include prevention of HIV in all women; prevention of unintended pregnancy among HIV infected women, preventing HIV infected mothers from passing on infection to their new born. Voluntary counseling and testing (VCT) was used to identifying HIV infected and uninfected mothers, single dose of antiretroviral prophylaxis, improving obstetric practice and counseling on infant feeding options and finally care and support for HIV infected women, their children and families. ${ }^{4}$
Some developing countries have already succeeded in providing PMTCT services to most HIV positive pregnant women who attend antenatal clinics. Results from these studies reported less than $4 \%$ of babies born to HIV positive mothers in developing countries becoming HIV positive, a finding that was comparable with the findings from USA and Western Europe. ${ }^{5}$ Although third generation antibody based tests such as ELISA are still commonly used to diagnose HIV in children, the ELISA requires a lead period of up to 18 months of age before the maternal HIV sero-status can be distinguished from that of the child. In developed countries, fourth generation antigen based tests using PCR have proven to be a viral tool in early diagnosis of the disease and thus narrowing the window period to less than one month. ${ }^{6}$ Nonetheless the antibody based tests are still an invaluable proxy of the antigen based tests in resource poor countries.

The majority of uninfected non-breast fed children will seroconvert by age of 15 months, but a smaller percentage (1\%-18\%) will not revert until age 18 months. ${ }^{7}$

Despite these limitations, HIV ELISA and rapid tests are the most widely available tests, and provide (or exclude) evidence of exposure. ${ }^{8}$

PMTCT was introduced in Edaga Hamus Hospital in Eritrea as a pilot project in 2002. The program was extended to Zoba Mekeal and Debub in 2004. Currently the practice of PMTCT is the operational government policy. ${ }^{9}$

This study was done to assess the effect of PMTCT by comparing the data before the start of PMTCT in 
selected health facilities in Eritrea.

\section{Methods}

The study had two components; a retrospective and a prospective component. The retrospective study was conducted using medical records of children born to mothers who were HIV positive prior to the introduction of PMTCT from Keren Hospital, one of the six regional referral hospitals in Eritrea. Medical records of all pregnant women who were HIV positive were compiled and analyzed.

The prospective study was conducted in Keren Hospital and in two other health facilities in Asmara, the Capital City of Eritrea; Orotta National Referral Pediatric Hospital and Feleg Hewet Health center, Asmara, Eritrea.

All pregnant women who were HIV positive at the three health facilities were enrolled in the PMTCT as per WHO guidelines adopted for use in Eritrea. All study subjects signed informed consent prior to the study. According to the guidelines VCT was done to all the pregnant women following the antenatal care. Information that was documented and analyzed was related to HIV transmission and PMTCT in particular, the mode of transmission and methods of PMTCT of HIV using safe deliveries, use of antiretroviral drugs and the baby feeding options. All the HIV positive pregnant women gave birth in the hospital. At onset of labour single dose of Nevirapine 200mg was given to every woman and $2 \mathrm{mg} / \mathrm{kg}$ to their babies within one to two days following delivery. All the infants were given cotrimoxazole $5 \mathrm{mg} / \mathrm{kg} /$ day from the age of six weeks until they were established to be HIV negative. Children were followed for 18 months and all of them were clinically healthy and blood samples were taken for ELISA.

This study was conducted from April 2006 to May 2008 after approved by the Ministry of Health of Eritrea. The main outcome measure of the study was the HIV serology status using the ELISA antibody based test done at the age of 18 months.

\section{Results}

Retrospective study before PMTCT in Keren Regional Referral Hospital

Out of the 33 babies born to mothers who were HIV positive followed up in Keren Hospital, nine were HIV positive at 18 months of age representing $27 \%$ of the children at risk of vertical transmission.

\section{Combined data for the three prospective data sources}

Of the 105, which was the total number of babies born to HIV positive mothers who participated in the PMTCT in the three health centres and underwent HIV ELIZA test at 18 months of age, only 5 babies were HIV positive, representing $4.7 \%$ of all children at risk of vertical transmission (Figure1).

Figure 1: HIV status among children before and after PMTCT in Eritrea

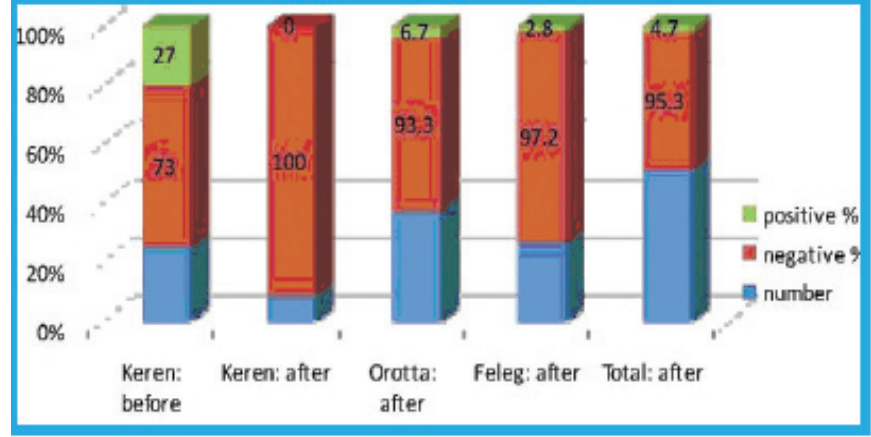

Prospective studies after PMTCT at Orotta National Referral Pediatric Hospital

Sixty children born to mothers who were HIV positive participated in the PMTCT program. Four children were HIV positive when tested at 18 months of age representing $6.7 \%$ of all the babies at risk of vertical transmission. For these four children who sero-converted, both the mothers and the babies received ARV and prophylactic cotrimoxazole as per guidelines.

One mother/baby pair did not receive the ARV prophylaxis while another 4 babies did not receive prophylactic cotrimoxazole. In spite of the critical omissions the 5 children were HIV negative.

\section{Prospective study at Feleg Hewet Health Center, Asmara}

Out of the thirty six babies born to HIV positive mothers who participated in PMTCT program, only one child sero-converted at 18 months of age, representing $2.8 \%$ of the children at risk of vertical transmission. This one child was delivered by cesarean section for delayed labor and premature rupture of membranes and artificially fed (Table 1).

\begin{tabular}{|c|c|c|c|c|c|c|}
\hline \multirow{2}{*}{$\begin{array}{l}\text { G } \\
\text { e } \\
n \\
d \\
\text { e } \\
r\end{array}$} & \multirow{2}{*}{$\begin{array}{l}\mathrm{N} \\
\mathrm{u} \\
\mathrm{m} \\
\mathrm{b} \\
\mathrm{e} \\
\mathrm{r}\end{array}$} & \multicolumn{2}{|c|}{ Mode of delivery } & \multicolumn{2}{|c|}{$\begin{array}{l}\text { Feeding } \\
\text { pattern }\end{array}$} & \multirow{2}{*}{\begin{tabular}{|l} 
Rec \\
eived \\
pro \\
phy \\
laxis
\end{tabular}} \\
\hline & & $\begin{array}{l}\text { Normal } \\
\text { vaginal }\end{array}$ & $\begin{array}{l}\text { Caes } \\
\text { arian } \\
\text { sec } \\
\text { tion }\end{array}$ & $\begin{array}{l}\text { Exc } \\
\text { lusive } \\
\text { breast } \\
\text { feed } \\
\text { ing } \\
\end{array}$ & $\begin{array}{l}\text { Arti } \\
\text { ficial } \\
\text { feed } \\
\text { ing }\end{array}$ & \\
\hline Female & 22 & 18 & 4 & 21 & 1 & 20 \\
\hline Male & 14 & 13 & 1 & 8 & 6 & 12 \\
\hline Total & 36 & 31 & 5 & 29 & 7 & 32 \\
\hline
\end{tabular}

Prospective study at Keren Hospital

All nine babies born to HIV mothers at Keren Hospital after the introduction of PMTCT were HIV negative after 18 months of age. All the babies were delivered vaginally, either exclusively breast fed for up to 4 months (2/9) or artificially fed (7/9).

\section{Discussion}

The impact of PMTCT in Eritrea was assessed using a retrospective study that examined all records of babies 
born to HIV positive mothers prior to PMTCT at Keren Hospital as well as prospective data generated from babies born to HIV positive mothers who participated in PMTCT following its introduction. ELISA was used for diagnosis of HIV at 18months of the child's age. Prior to PMTCT, sero-conversion was $27 \%$ compared to less than $5 \%$ after PMTCT. The authors conclude that the remarkable impact of PMTCT in Eritrea could be increased if skilled delivery attendances were improved that can facilitate participation of HIV positive pregnant women in PMTCT, thus reducing risk of peripartum transmission of the disease and consequently reducing childhood morbidity and mortality.

The relatively small sample sizes in the study could be explained by the low HIV prevalence of less than 2.4\% in Eritrea based on sentinel surveys and the rate is beginning to plateau. ${ }^{9}$ Another possible contributing factor is the low skilled care delivery attendance of about $30 \%$ in spite of a high antenatal care visit of at least more than $70 \% \cdot{ }^{10}$

Seroconversion was determined using ELISA an antibody based test rather than the antigen based PCR because of limited availability, and expertise resources including human expertise in our resource limited setting. HIV testing using ELISA is a good proxy of PCR and gives equivalent results to PCR provided it is conducted after the age of 18 months. ${ }^{8}$

The pre-PMTCT vertical transmission rate of HIV reported here falls within the range from 25 to $40 \%$ previously observed in other reports where case/ control studies were ethically acceptable prior to availability of empirical evidence for unquestionable efficacy of PMTCT. ${ }^{11}$

Vertical transmission is one of the most important routes of HIV-1 transmission in sub-Saharan Africa being responsible for more than $5 \%$ of all HIV/AIDS transmission globally with the biggest burden in Southern Africa. ${ }^{4}$ Because of the simplicity of its application the low cost of the regimen, PMTCT has extensive potential to decimate the HIV prevalence rates among children. Many studies have advocated for the scaling up of the practice to cover various countries and indeed whole world. There are some obstacles to be managed in the process such as resistance to being tested in the first place, low antenatal care and skilled delivery attendances, quality of antenatal care and delivery issues like episiotomies in primiparous women, the whole issue of stigma, non compliance with developed countries have already circumvented and addressed some of these obstacles by making HIV testing a routine antenatal care investigation, combination ARV drugs, elective cesarean section and complete avoidance of breast feeding have resulted into mother to child transmission of less than $2 \% .{ }^{5}$

Although this study has some limitations such as the small sample sizes, the incompleteness of the retrospective data pertaining to the maternal factors, the authors have demonstrated that the PMTCT program is effective in reducing the mother to child transmission. Expansion of the program and making HIV testing mandatory for all pregnant women as well as increasing antenatal and skilled care deliveries, childhood morbidity and mortality can be minimized to levels reported in developed countries or lower.

\section{Acknowledgments}

The authors are grateful to the Ministry of Health, State of Eritrea; Orotta School of Medicine, Asmara, Eritrea; Research and Human Resource Development of Ministry of Health, Eritrea; and NATCOD particularly the PMTCT UNIT. Last but not least thanks to all the mothers, children involved in this study and to all my colleagues who suggested an idea in my study.

\section{References:}

1. De Cock K.M. et al. "Prevention of mother-to-child HIV transmission in resource-poor countries: translating research into policy and practice", JAMA 2000; 283:11751182.

2. Temmerman M. et al, "Mother-to-child HIV transmission in resource poor settings: how to improve coverage? AIDS 2003; 17: 1239-1242

3. Simab D, Kamwela J, Mpembemni R, Msamanga G. The impact of scaling-up prevention of mother to child transmission of HIV infection on the human resource requirement: the need to go beyond numbers. Int J health Plann Manage. 2008 (Epub ahead of print).

4. Spensley A, sripipatanaT,Turner An, Hoblitzeelle C, Robinson J, Wilfert C, Prevention of Mother To Child transmission of HIV Group TE. Preventing mother to child transmission of HIV in Resource Limited settings: The Elizabeth Glaser Pediatric AIDS Foundation Experience. Am J Pub Health. 2008; Epub ahead of print.

5. Read JS; Committee on Pediatric AIDS, American Academy of Diagnosis of HIV-1 infection in children younger than 18 months in the United States Pediatrics. Pediatrics. 2007;120: e1547-62

6. Chi BH, Sinkala $M$, Stringer EM, Cantrell RA, Mtonga $V$, Bulterys M, Zulu I, Kankasa C, Wilfert C, Weidle PJ, Vermund $\mathrm{SH}$, Stringer JS. Early clinical and immune response to NNRTI-based antiretroviral therapy among women with prior exposure to single dose nevirapine. AIDS. 2007; 21: 957-64.

7. Onankpa B, Airede L, Paul I, Dorcas I. Pattern of pediatric HIV/AIDS: a five experience in a tertiary hospital. J Natl Med Assoc. 2008;100:821-5

8. Costagliola D, Damond F, Palmer P, Rouzioux C, BrunVezinet F. One or two enzyme-linked immunosorbent assay tests on the first serum sample for initial diagnosis of HIV-1 infection? AIDS. 2008; 22: 2042-4.

9. Ministry of Health, Eritrea, PMTCT Unit Annual report 2007.

10. Ministry of Health, Eritrea, Annual Report 2007.

11. Kumar RM, Uduman SA, Khurranna AK. A prospective study of mother to infant HIV transmission in tribal women from India. J Acquir Immune Defic Syndr Hum Retrovirol. 1995;9: 238-42. 